\title{
Fatty acid metabolic enzyme acyl-CoA thioesterase 8 promotes the development of hepatocellular carcinoma
}

\author{
YU-HSUAN HUNG ${ }^{1,2 *}$, YI-SHIN CHAN ${ }^{2 *}$, YUNG-SHENG CHANG $^{1,2}$, KUO-TING LEE $^{3}$, HUI-PING HSU ${ }^{3}$ \\ MENG-CHI YEN ${ }^{1,2}$, WEI-CHING CHEN ${ }^{1,2}$, CHIH-YANG WANG ${ }^{1,2}$ and MING-DERG LAI ${ }^{1,2,4}$ \\ ${ }^{1}$ Institute of Basic Medical Sciences; Departments of ${ }^{2}$ Biochemistry and Molecular Biology, and ${ }^{3}$ Surgery; \\ ${ }^{4}$ Center for Infectious Diseases and Signaling Research, College of Medicine, \\ National Cheng Kung University, Tainan, Taiwan, R.O.C.
}

Received February 7, 2014; Accepted March 20, 2014

DOI: $10.3892 /$ or.2014.3155

\begin{abstract}
Dysregulated metabolism is an emerging hallmark of cancer development, and upregulated lipid synthesis is one of the important tumor metabolic features. However, lipolysis may also contribute to cancer pathogenesis by altering free fatty acid (FFA) metabolism. In the present study, we investigated the importance of the lipolytic enzyme acyl-CoA thioesterase 8 (ACOT8) in hepatocellular carcinoma (HCC) development. Bioinformatic analysis of published microarrays regarding clinical specimens revealed that both ACOT8 gene copy number and mRNA expression were increased in HCC tissues when compared to these variables in nontumor tissues. ACOT8 silencing with specific shRNA stably expressed in Huh7 and Hep3B HCC cell lines showed that ACOT8 protein expression and overall thioesterase activity were reduced following ACOT8 knockdown. In vitro tumorigenic assays revealed that ACOT8 knockdown inhibited anchorage-dependent and -independent growth of HCC cell lines. This growth inhibition was partially rescued by addition of the FFA, myristic acid, indicating the importance of FFA in cancer metabolism. In summary, lipolytic enzyme ACOT8 is frequently upregulated in HCC clinical specimens. More importantly, ACOT8 silencing leads to inhibition of cell growth in HCC in vitro.
\end{abstract}

Correspondence to: Professor Ming-Derg Lai, Department of Biochemistry and Molecular Biology, College of Medicine, National Cheng Kung University, Tainan, Taiwan, R.O.C.

E-mail: a1211207@mail.ncku.edu.tw

*Contributed equally

Abbreviations: HCC, hepatocellular carcinoma; ACOT, acyl-CoA thioesterase; FFA, free fatty acid; CoA, coenzyme A

Key words: acyl-CoA thioesterase 8, fatty acid metabolism, hepatocellular carcinoma

\section{Introduction}

Hepatocellular carcinoma (HCC) is the fifth leading cause of cancer-related mortality in the world $(1,2)$. The high recurrence rate and poor prognosis of HCC are responsible for its high mortality. Even though surgery, locoregional therapy, transcatheter arterial chemoembolization (3) and chemotherapy are available for the treatment of HCC, they have offered limited success in reducing cancer-related mortality. Target therapy with the multiple tyrosine kinase inhibitor sorafenib has demonstrated improved therapeutic potential for HCC patients and has prolonged their survival (4), yet more therapeutic targets are awaiting identification for HCC.

Among the multiple hallmarks of tumor development, dependence on glycolysis is one of the major characteristics in various types of cancers including HCC (5-7), and it is not only a critical issue in cancer research but also serves as a therapeutic target for cancer treatment (8-10). In addition to glucose metabolism, cancer cells also remodel the metabolism of many macromolecules, including amino acids $(6,11)$ and fatty acids $(12-14)$, to support the formation of neoplasia. We previously demonstrated that dysregulation of argininosuccinate lyase (ASL) (15) and long-chain acyl-CoA synthetase (ACSL) (16) contributes to the development of HCC. Fatty acid serves as one of the major energy providers for cellular metabolism, and its involvement in tumor development has been demonstrated by the upregulation of fatty acid synthase (FASN) during cancer formation, and the expression of FASN even correlates with increased disease progression and decreased patient survival (14). However, recent studies also revealed that lipolysis plays a critical role in cancer pathogenesis. In human multiple myeloma cancer cells, inhibition of fatty acid degradation was found to reduce proliferation (13). In various types of cancers (17) including colorectal cancer (18), silencing of the lipolysis-related enzyme monoacylglycerol lipase (MAGL) was found to reduce tumor formation. In lipolysis-related enzyme acyl-CoA thioesterase superfamily member 1 (ACOT11) knockout mice, the animals were protected against disease-prone factors, such as obesity and insulin resistance (19). Therefore, production of fatty acid by 
either de novo synthesis or by reduction in its degradation is important in cancer development (20-22).

Among the lipolysis-related enzymes, members of the acyl-CoA thioesterase (ACOT) superfamily control an important step in lipid utilization by catalyzing the breakdown of fatty acyl-CoA into free fatty acid (FFA) and coenzyme A (CoA) molecule. To date, 15 ACOTs have been identified and are divided according to structure into two superfamilies; the first one is the $\alpha / \beta$-hydrolase superfamily which includes ACOT1-6, and the second one is hotdog-fold protein superfamily which includes ACOT7-15. Different ACOTs display different specificity toward fatty acyl-CoA of different chain length and reside in different cellular compartments (23-25). Studies addressing the functions of distinct ACOTs by means of transgenic mice including ACOT7 $7^{-/}(26)$, ACOT11 ${ }^{-/}(19,27)$ and $\mathrm{ACOT}_{13}{ }^{-/}(28,29)$ revealed that different ACOTs may have diverse functions in regulating lipid metabolism. Among these ACOTs, ACOT8 reacts with a wide range of substrates, including short-, medium- and long-chain fatty acids (25), implying its essential role in lipid utilization and regulation of lipid metabolism for fatty acids of nearly all chain lengths. Therefore, dysregulation of ACOT8 is expected to have a strong association with human disease. Indeed, ACOT8 is associated with ovarian cancer and lung adenocarcinoma. Increased ACOT8 gene copy number has been observed in clinical specimens from patients with ovarian cancer (30), and high ACOT8 expression is associated with metastasis as well as poor outcome in patients with lung adenocarcinoma (31). However, the importance of ACOT8 in HCC, which is one of the cancer types seriously impacted by dysregulated lipid metabolism, has not yet been investigated. In the present study, we first identified the importance of ACOT8 during HCC formation by performing bioinformatic analysis of published microarrays with HCC clinical specimens in the GEO database (32), and found that ACOT8 gene copy number and mRNA expression were increased in HCC tissues when compared to these variables in non-tumor tissues. We then knocked down ACOT8 expression in two HCC cell lines, and observed a decrease in anchorage-dependent and -independent growth in the cancer cells. The reduction in cell growth was compensated by reintroduction of ACOT8 or partially recovered by the addition of FFA myristic acid. Collectively, ACOT8 plays an important role in the formation of $\mathrm{HCC}$, and downregulation of ACOT8 inhibits the growth of tumor cells.

\section{Materials and methods}

Data collection of microarrays regarding human $\mathrm{HCC}$ clinical specimens. Microarrays regarding human HCC clinical specimens published from 2004 to 2013 were collected from the public resource NCBI Gene Expression Omnibus (GEO, http://www.ncbi.nlm.nih.gov/geo). Microarrays regarding HCC cell lines were not included. Four microarray datasets GSE14322 (33), GSE1898 (34), GE25097 (35) and GSE10140-GSE10141 (36) were collected, and the change in ACOT8 gene copy number and mRNA expression in these microarrays was significantly different in the HCC tissues when compared to these variables in the non-tumor tissues. We downloaded the normalized signals for each microarray directly from NCBI Gene Expression Omnibus, and these normalized signals were further analyzed to identify the tendency of the change in ACOT8 gene copy number or mRNA expression in $\mathrm{HCC}$ tissues. The result of this analysis was assessed for statistical significance and diagrammed using GraphPad Prism, version 5 (GraphPad Software, San Diego, CA, USA). The detailed information of these microarrays is listed in Table I.

Cell lines. Human HCC cell line Huh7 was kindly provided by I.J. Su of the National Health Research Institute. Huh7 and another human HCC cell line Hep3B as well as their shACOT8 stable transfectant clones were cultured in DMEM containing 10\% FBS (Biological Industries, Beit Haemek, Israel) and $1 \%$ penicillin-streptomycin. Cells were kept in an incubator at $37^{\circ} \mathrm{C}$ with $5 \% \mathrm{CO}_{2}$.

Chemicals, reagents, plasmids and antibodies. Ethidium bromide, sodium dodecyl sulfate (SDS), G418, myristic acid, myristoyl-CoA lithium salt, and 5,5-dithiobis-(2-nitrobenzoic acid) (DTNB) were purchased from Sigma (St. Louis, MO, USA). The Micro $\mathrm{BCA}^{\mathrm{TM}}$ protein assay reagent kit was purchased from Pierce (Woburn, MA, USA). DMEM and an antibiotic mixture were purchased from Invitrogen (Carlsbad, CA, USA). Turbofect transfection reagent was purchased from Fermentas (Glen Burnie, MD, USA). Antibodies against ACOT8 (NBP1-66562; Novus Biologicals, Littleton, CO, USA) and tubulin (GTX628802; GeneTex, Taiwan) were used in the western blot analysis.

RNA interference. The shACOT8 plasmid-carrying bacterial clone was obtained from the RNAi core facility (Academia Sinica, Taiwan), and the target sequence used was 5'-CAT TGGCGCTCAACCGAATTG-3'. Cells were transfected with shRNA against luciferase as vector control or against ACOT8 and selected for stable transfectant clones with puromycin.

Plasmid construction. Human ACOT8 (NM_005469.3) was constructed into the HA6L vector as previously described (37). Cells were transfected with the ACOT8-overexpression plasmid and selected for stable transfectant clones with G418. The expression of ACOT 8 in these clones was confirmed at the protein level.

Western blot analysis. Cells were lysed in modified RIPA buffer with protease inhibitors and let stand on ice for $20 \mathrm{~min}$. After centrifugation at $12,000 \mathrm{rpm}$, at $4^{\circ} \mathrm{C}$ for $10 \mathrm{~min}$, the supernatant was harvested and the protein concentration was assayed using the Micro BCA protein assay reagent kit. Samples with the same amount of protein and $4 \mathrm{X}$ sample buffer were mixed, heated at $95^{\circ} \mathrm{C}$ for $5 \mathrm{~min}$, and subjected to electrophoresis. The protein was then transferred onto a PVDF membrane (Millipore, Bedford, MA, USA) using the Hoefer Semiphor Semi-Dry transfer unit (Amersham Pharmacia, San Francisco, CA, USA), and blocked in 5\% non-fat milk at room temperature for $1 \mathrm{~h}$. Specific antibodies were added to the probe targets overnight at $4^{\circ} \mathrm{C}$. Following washing with $0.1 \%$ TBS-T for $10 \mathrm{~min}$ for 3 times, the membrane was probed with the specific secondary antibody at room temperature for $1 \mathrm{~h}$ and washed with $0.1 \%$ TBS-T. The quantity of target proteins and internal control were identified by adding chemiluminescence 
Table I. Characteristics of the collected GEO Datasets.

\begin{tabular}{lccrr}
\hline GEO accession No. & References & Type of microarray & No. of tumors & No. of normal DNA \\
\hline GSE14322 & Roessler, et al (33) & Genome variation & 76 & $-^{\mathrm{a}}$ \\
GSE1898 & Lee, et al (34) & Expression & 91 & 18 \\
GSE25097 & Tung, et al (35) & Expression & 268 & 243 \\
GSE10140, GSE10141 & Hoshida, et al (36) & Expression & 80 & 82 \\
\hline
\end{tabular}

${ }^{a}$ Normal DNA from Promega was applied.



Figure 1. Increased ACOT8 gene copy number in HCC. GSE14322 microarray from the GEO database was analyzed for ACOT8 gene copy number in genomic DNA from HCC specimens with normalization using normal DNA.

reagent ECL (Millipore) onto the membrane and the luminescent intensity was recorded using the BioSpectrum AC imaging system (UVP, Upland, CA, USA).

Thioesterase activity assay. The thioesterase activity assay was performed according to a previous report (19) with some modifications. Total protein was harvested from cells in homogenization buffer (1\% Tergitol NP-40, $0.5 \%$ sodium deoxycholate, $0.1 \%$ SDS, $150 \mathrm{mM} \mathrm{NaCl}, 50 \mathrm{mM}$ Tris- $\mathrm{HCl}$ and $2 \mathrm{mM}$ EDTA) and was allowed to remain in ice for $20 \mathrm{~min}$. After centrifugation at $12,000 \mathrm{rpm}$ at $4^{\circ} \mathrm{C}$ for $10 \mathrm{~min}$, the supernatant was harvested and the protein concentration was assayed using the Micro BCA protein assay reagent kit. Protein at the indicated amount was added, and the reaction volume was adjusted with the reaction buffer $[50 \mathrm{mM} \mathrm{KCl}$, $10 \mathrm{mM}$ HEPES (pH 7.5) and $0.3 \mathrm{mM}$ DTNB] to a final volume of $200 \mu \mathrm{l}$. Myristoyl-CoA at the final concentration of $200 \mathrm{nM}$ was then added to start the thioesterase activity assay. Thioesterase activity was analyzed at 5-min intervals for a total of $1 \mathrm{~h}$ with the ELISA reader at a wavelength of $405 \mathrm{~nm}$.

Colony formation assay. A total of $5 \times 10^{2}$ Huh7 cells, $1 \times 10^{3}$ Hep3B cells and same amount of cells for their stable transfectant clones of ACOT8 knockdown or compensation were seeded onto 6-well plates and cultured for 9 days to assay the anchorage-dependent growth ability of the cancer cells. The number of colonies was identified by methyl blue staining, counted under a microscope and analyzed.

Soft agar assay. Cells $\left(5 \times 10^{3}\right)$ were seeded into $1 \mathrm{ml}$ $0.3 \%$ agar-containing medium and then onto $0.6 \%$ agar- covered 6-well plates and cultured for 14 days to assay the anchorage-independent growth ability of the cancer cells. The number of colonies was identified by crystal violet staining, counted under a microscope and analyzed.

Statistical analysis. All statistical analyses were performed by the GraphPad Prism, version 5 (GraphPad Software, San Diego, CA, USA). All error bars in the figures represent SEM. Student's t-test and two-way ANOVA followed by Bonferroni post-test were used for analysis of difference between each experimental group, and a P-value of $<0.05$ was considered to indicate a statistically significant difference.

\section{Results}

Bioinformatic analysis of the ACOT8 gene copy number and $\mathrm{mRNA}$ expression in the HCC clinical specimens. To investigate the importance of ACOT8 dysregulation in HCC tumorigenesis in terms of human cancer patients, we performed bioinformatic analysis of the published microarray data with HCC tissues and non-tumor tissues from the GEO database (32). The analysis revealed that ACOT8 gene copy number was increased in the HCC tissues when compared to that in the normal DNA in microarray GSE14322 (Fig. 1) from the US, since 64 of the 76 HCC patients in GSE14322 (33) displayed increased ACOT8 gene copy number than that of normal DNA. In addition, ACOT8 mRNA expression was significantly increased in HCC tissues compared to the expression in the non-tumor tissues in microarrays GSE1898 (Fig. 2A), GSE25097 (Fig. 2B) and GSE10140-GSE10141 (Fig. 2C). The above phenomena were observed in 73 of 91 HCC patients in 
A
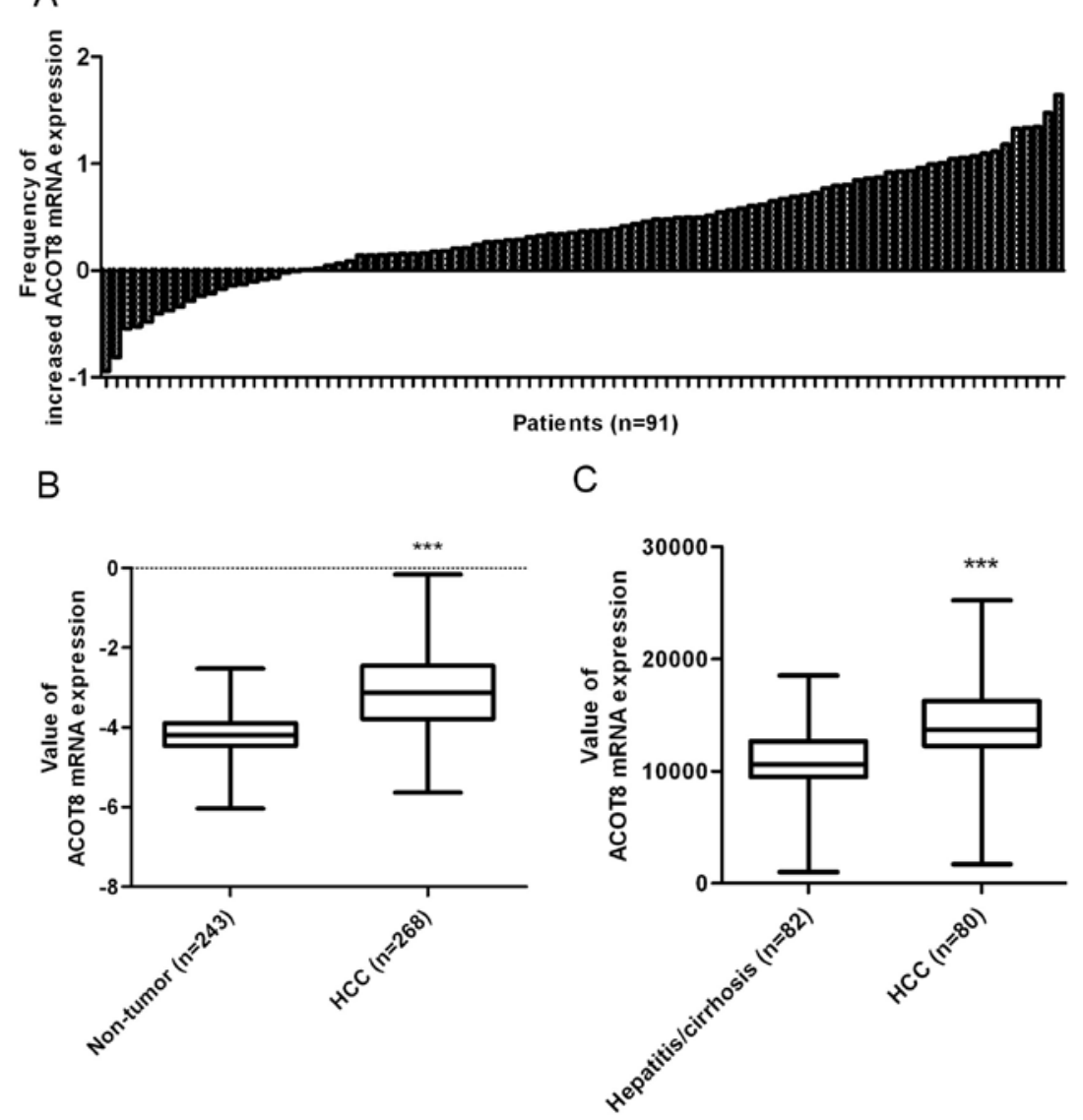

Figure 2. Increased ACOT8 mRNA expression in HCC. (A) GSE1898 microarray from the GEO database was analyzed for ACOT8 mRNA expression in liver tumor specimens with normalization using RNA from non-tumor liver specimens. (B) GSE25097 microarray from the GEO database was analyzed for ACOT8 mRNA expression in adjacent non-tumor and liver tumor specimens. (C) GSE10140 and GSE10141 microarrays from the GEO database were analyzed for ACOT8 mRNA expression in adjacent non-tumor and liver tumor specimens. Error bars represent SEM. Statistical difference between ACOT8 mRNA expression in adjacent non-tumor and liver tumor specimens was examined $\left({ }^{* * *} \mathrm{P}<0.0001\right)$.

GSE1898 (34), 268 HCC tissues vs. 243 non-tumor tissues in GSE25097 (35) and 80 HCC tissues vs. 82 non-tumor tissues in GSE10140-GSE10141 (36). This bioinformatic analysis revealed that the dysregulation in ACOT8, particularly upregulation in both gene copy number and mRNA expression, is associated with $\mathrm{HCC}$ development in respect to human cancer patients.

ACOT8 modulates in vitro tumorigenesis and thioesterase activity in HCC. To study the contribution of ACOT8 expression during HCC formation in detail, shRNA against ACOT8 was transfected into the Huh7 and Hep3B HCC cell lines, and stable transfectant clones were established. Knockdown efficiency was confirmed at the protein level (Fig. 3A and B). The ACOT8 shRNA stable transfectants were then subjected to in vitro tumorigenic and thioesterase activity assays to determine the role of ACOT8 in HCC development. Knockdown of ACOT8 caused a significant decrease in anchorage-dependent (Fig. 3C and D) and anchorage-independent growth (Fig. 3E and F) in both HCC cell lines, indicating that ACOT8 silencing reduced the in vitro tumorigenicity of HCC. We also investigated the effect of ACOT8 knockdown on thioesterase activity to determine the possible involvement of the lipolytic role of ACOT8 during HCC formation. The alteration in thioesterase activity was measured by the change in absorbance at $405 \mathrm{~nm}$ resulting from the reaction between ACOT and DTNB. The ACOT-mediated hydrolysis of fatty acyl-CoA and the release of free CoA molecules lead to a rapid reaction between DTNB and CoA, giving rise to the production of 5-thio-2-nitrobenzoate, whose absorbance can be detected at $412 \mathrm{~nm}$ (19) or $405 \mathrm{~nm}$ (present study). Indeed, the ACOT8knockdown clones displayed a reduced absorbance value and thus reduced thioesterase activity when compared to that of the vector control clones (Fig. 4A and B), indicating ACOT8 plays a major role in total lipolysis. To further exclude the off-target effect of ACOT8 shRNA in the above experiments, ACOT8 was reintroduced into the ACOT8-knockdown clones in Hep3B cells (Fig. 5A), and the cell growth was restored as determined by a colony formation assay (Fig. 5B), further supporting the important role of ACOT8 in HCC formation.

ACOT8-mediated HCC tumorigenesis is dependent on the FFA level. To elucidate the metabolic mechanisms involved in ACOT8-modulated HCC development, we examined the effect of FFA metabolites on the ACOT8 knockdown-reduced tumorigenesis. One of the metabolic products downstream of ACOT8-mediated reaction, the FFA myristic acid, was added to the medium to ascertain whether FFA affects the growth inhibition induced by ACOT8 knockdown. The addition of myristic acid indeed led to the increased growth of cancer 
A

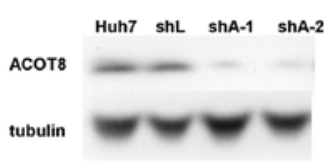

B

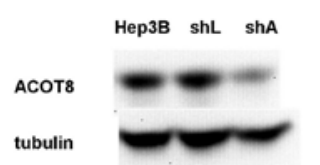

C

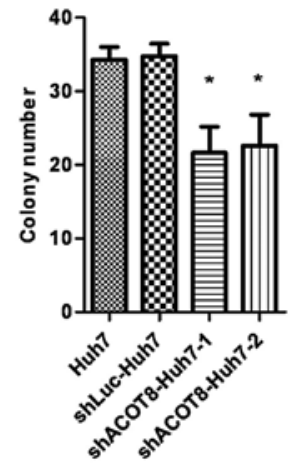

D

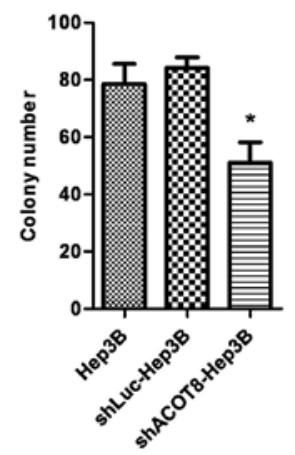

E

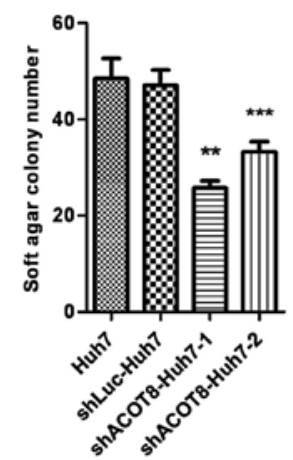

F

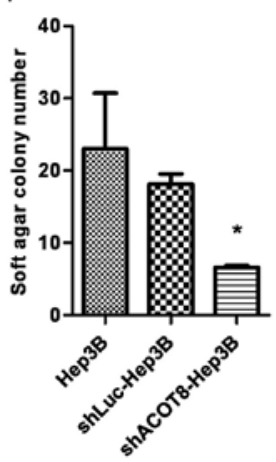

Figure 3. ACOT8 shRNA reduces in vitro HCC tumorigenesis. (A and B) The knockdown efficiency of shRNA against ACOT8 (shA) in Huh7 and Hep3B cells compared to that of shRNA against luciferase (shL) was determined by western blotting. Tumorigenic ability of the indicated clones was identified by anchorage-dependent growth colony formation assay in (C) Huh7 and (D) Hep3B cells, respectively, as well as by anchorage-independent growth soft agar assay in (E) Huh7 and (F) Hep3B cells, respectively. Error bars represent SEM. Statistical difference in anchorage-dependent and anchorage-independent growth ability between shLuc and shACOT8 clones was examined $\left({ }^{*} \mathrm{P}<0.05,{ }^{* * *} \mathrm{P}<0.01,{ }^{* * * *} \mathrm{P}<0.001\right)$.
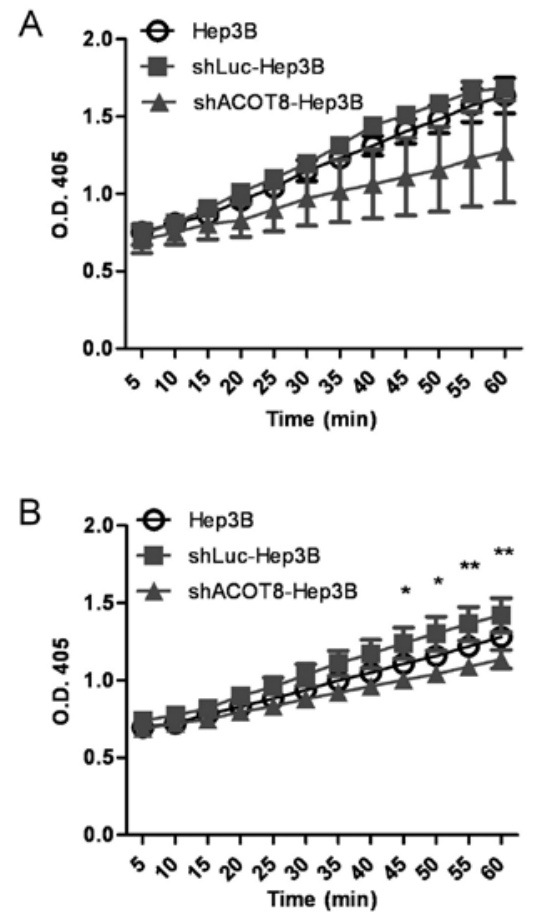

Figure 4. ACOT8 shRNA reduces thioesterase activity. Total protein was harvested in homogenization buffer and (A) $6.25 \mu \mathrm{g}$ or (B) $3.125 \mu \mathrm{g}$ of protein was prepared in reaction buffer and then subjected to thioesterase activity assay at the time point indicated. Error bars represent SEM. Statistical difference in thioesterase activity between shLuc-Hep3B and shACOT8-Hep3B was examined by two-way ANOVA followed by Bonferroni post-test $\left({ }^{*} \mathrm{P}<0.05,{ }^{* *} \mathrm{P}<0.01\right)$.
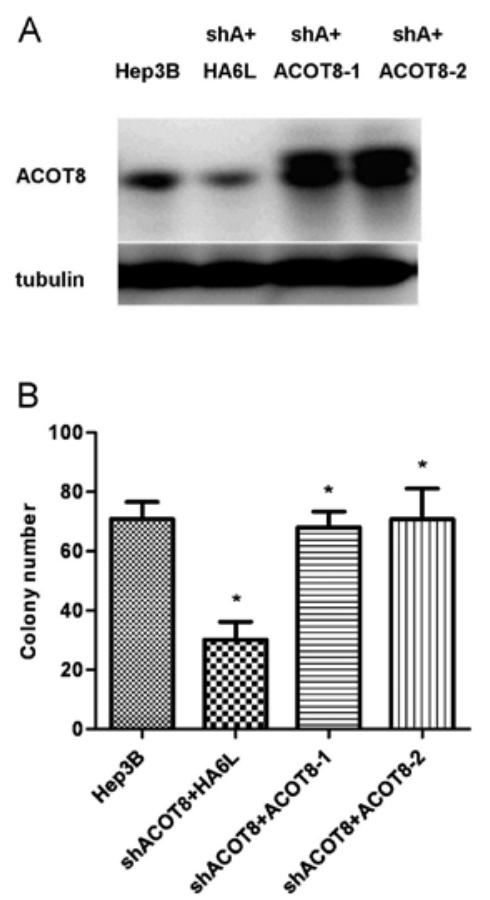

Figure 5. ACOT8 shRNA-mediated growth inhibition is restored by ACOT8 compensation. (A) ACOT8 shRNA stable transfectants were reintroduced with vector HA6L or ACOT8 in Hep3B, and the compensation efficiency was determined by western blotting. (B) Tumorigenic ability of the indicated clones was identified by anchorage-dependent growth colony formation assay. Error bars represent SEM. Statistical difference in anchorage-dependent growth ability between i) Hep3B and vector control HA6L clone as well as ii) HA6L clone and ACOT8 compensation clones was examined ( $\mathrm{P}<0.05)$. 



Figure 6. ACOT8 shRNA-mediated growth inhibition is partially restored by addition of myristic acid. ACOT8 downstream metabolite myristic acid was used to treat (A) shACOT8-Huh7 and (B) shACOT8-Hep3B, and its effect on cancer cell growth was analyzed. Various doses of myristic acid were repeatedly added at 3-day intervals during refreshing of medium, and the number of colonies was determined 9 days later. P in A indicates shACOT8-Huh7 without DMSO addition. Error bars represent SEM. Statistical difference between the DMSO vehicle group and the myristic acid treatment group was examined $\left({ }^{*} \mathrm{P}<0.05,{ }^{* *} \mathrm{P}<0.01\right)$.

cells as determined by a colony formation assay in ACOT8knockdown stable transfectants of Huh7 and Hep3B HCC cell lines (Fig. 6A and B). The effects of myristic acid on restoration of the cell growth were more prominent in the Hep3B stable transfectants. This result revealed that the metabolite downstream of the ACOT8-mediated reaction was able to rescue ACOT8 knockdown-decreased HCC cell growth, further supporting the notion that lipolysis enzyme ACOT8 plays an important role in HCC development.

\section{Discussion}

In the present study, we identified the importance of lipolysis in HCC development by determining the contribution of an acyl-CoA degrading lipolytic enzyme ACOT8 in HCC formation. ACOT is a family of enzymes controlling one of the critical steps in lipid utilization by catalyzing the breakdown of fatty acyl-CoA into FFA and CoA molecules. ACOT8 is one of the family members with a wide range of substrates, which also indicates its possible involvement in disease progression or even cancer formation. Indeed, the participation of ACOT8 in ovarian cancer (30) and lung adenocarcinoma (31) has been reported in a clinical respect, while the impact of ACOT8 expression on other cancer types which are seriously influenced by dysregulated lipid metabolism such as HCC and whether the lipolytic role of ACOT8 participates in these processes remain to be elucidated. Aiming to identify the importance of ACOT8 in HCC tumorigenesis and the mechanism involved, we first investigated the expression pattern of ACOT8 in HCC patients. By performing bioinformatic analysis on published microarray data with HCC clinical specimens in the GEO database, we determined that both gene copy number and mRNA expression of ACOT8 were increased in HCC tissues compared to these variables in the non-tumor tissues, indicating the association between ACOT8 expression and HCC tumorigenesis in clinical specimens. To further ascertain the effect of ACOT8 expression on HCC tumorigenesis, we performed in vitro tumorigenic and thioesterase activity assays following ACOT8 knockdown in HCC cell lines. ACOT8 knockdown reduced not only anchorage-dependent and -independent growth but also thioesterase activity in the cancer cells, suggesting the importance of ACOT8 in modulating HCC development. ACOT8 compensation in shRNA-knockdown clones increased cancer cell growth, which further supports the critical role of ACOT8 in HCC formation. The involvement of lipid metabolism in ACOT8-modulated HCC tumorigenesis was further demonstrated by the rescue of cell growth via addition of FFA myristic acid, indicating ACOT8-modulated HCC formation is partially, if not totally, regulated by its lipolytic role.

Since lipid metabolism is one of the important characteristics in the formation of neoplasias, numerous studies have investigated the role of lipolytic enzymes in cancer development $(13,17,18,22)$. The functions of ACOT family members in normal physiology and diseases have also been studied $(23-29,31)$. In addition to findings that ACOT8 gene copy number and expression are increased, respectively, in ovarian cancer and lung adenocarcinoma $(30,31)$, ACOT2 and ACOT4 have also be suggested to participate in breast cancer due to their overexpression $(38,39)$, with the finding that ACOT2 modulates the production of prostaglandin in breast cancer cells (38). In the present study, myristic acid, the 14-carbon saturated fatty acid, was able to increase the cell growth of ACOT8 shRNA stable transfectants to the level comparable to that of the vector control. Saturated fatty acids have been reported to have pro-apoptosis effects on normal and cancer cells due to lipotoxicity, but they have also been shown to enhance production of the pro-inflammatory cytokine interleukin-8 (IL-8) to interrupt lipid metabolism (40) in hepatocytes and HCC cells. Saturated fatty acids have also been linked to the increased risk of developing prostate cancer in a Japanese cohort (41). These findings indicate that the diverse functions of saturated fatty acids operate in a cell context-dependent fashion, and our study which supports a possible tumorigenic role of saturated fatty acid in HCC development is in accordance with these reports.

In conclusion, we reported the importance of acyl-CoA degrading lipolytic enzyme ACOT8 in the development of HCC by demonstrating that its gene copy number and mRNA expression are increased in HCC patients, while its knockdown reduced both in vitro tumorigenesis and thioesterase activity in HCC cells. To the best of our knowledge, this is the first report regarding the contribution of ACOT8 in HCC development, implying the importance of lipolysis in HCC formation.

\section{Acknowledgements}

This study was supported by the grant to M.-D. Lai, NSC-1002325-B-006-008 from the National Science Council, Taiwan; 
NHRI-EX100-9927B1 from the National Health Research Institute, Taiwan; to Establish Centers of Excellence for Cancer Research in Taiwan, DOH101-TD-C-111-003 Department of Health, Executive Yuan, Taiwan, R.O.C.

\section{References}

1. El-Serag HB and Rudolph KL: Hepatocellular carcinoma: epidemiology and molecular carcinogenesis. Gastroenterology 132 2557-2576, 2007

2. Thorgeirsson SS and Grisham JW: Molecular pathogenesis of human hepatocellular carcinoma. Nat Genet 31: 339-346, 2002.

3. Bruix J, Sala M and Llovet JM: Chemoembolization for hepatocellular carcinoma. Gastroenterology 127: S179-S188, 2004.

4. Zhu AX: Development of sorafenib and other molecularly targeted agents in hepatocellular carcinoma. Cancer 112: 250-259, 2008

5. Kroemer G and Pouyssegur J: Tumor cell metabolism: cancer's Achilles' heel. Cancer Cell 13: 472-482, 2008.

6. Ward PS and Thompson CB: Metabolic reprogramming: a cancer hallmark even Warburg did not anticipate. Cancer Cell 21: 297-308, 2012.

7. Xu RH, Pelicano H, Zhou Y, et al: Inhibition of glycolysis in cancer cells: a novel strategy to overcome drug resistance associated with mitochondrial respiratory defect and hypoxia. Cancer Res 65: 613-621, 2005.

8. Pelicano H, Martin D, Xu RH and Huang P: Glycolysis inhibition for anticancer treatment. Oncogene 25: 4633-4646, 2006.

9. Vander Heiden MG: Targeting cancer metabolism: a therapeutic window opens. Nat Rev Drug Discov 10: 671-684, 2011.

10. Tennant DA, Durán RV and Gottlieb E: Targeting metabolic transformation for cancer therapy. Nat Rev Cancer 10: 267-277, 2010.

11. Locasale JW: Serine, glycine and one-carbon units: cancer metabolism in full circle. Nat Rev Cancer 13: 572-583, 2013.

12. Menendez JA and Lupu R: Oncogenic properties of the endogenous fatty acid metabolism: molecular pathology of fatty acid synthase in cancer cells. Curr Opin Clin Nutr Metab Care 9: 346-357, 2006

13. Tirado-Vélez JM, Joumady I, Sáez-Benito A, Cózar-Castellano I and Perdomo G: Inhibition of fatty acid metabolism reduces human myeloma cells proliferation. PloS One 7: e46484, 2012.

14. Menendez JA and Lupu R: Fatty acid synthase and the lipogenic phenotype in cancer pathogenesis. Nat Rev Cancer 7: 763-777, 2007.

15. Huang HL, Hsu HP, Shieh SC, et al: Attenuation of argininosuccinate lyase inhibits cancer growth via cyclin A2 and nitric oxide. Mol Cancer Ther 12: 2505-2516, 2013.

16. Chang YS, Tsai CT, Huangfu CA, et al: ACSL3 and GSK-3 $\beta$ are essential for lipid upregulation induced by endoplasmic reticulum stress in liver cells. J Cell Biochem 112: 881-893, 2011.

17. Nomura DK, Long JZ, Niessen S, Hoover HS, Ng SW and Cravatt BF: Monoacylglycerol lipase regulates a fatty acid network that promotes cancer pathogenesis. Cell 140: 49-61, 2010.

18. Ye L, Zhang B, Seviour EG, et al: Monoacylglycerol lipase (MAGL) knockdown inhibits tumor cell growth in colorectal cancer. Cancer Lett 307: 6-17, 2011.

19. Zhang Y, Li Y, Niepel MW, et al: Targeted deletion of thioesterase superfamily member 1 promotes energy expenditure and protects against obesity and insulin resistance. Proc Natl Acad Sci USA 109: 5417-5422, 2012.

20. Nieman KM, Kenny HA, Penicka CV, et al: Adipocytes promote ovarian cancer metastasis and provide energy for rapid tumor growth. Nat Med 17: 1498-1503, 2011.

21. Kaini RR, Sillerud LO, Zhaorigetu S and Hu CA: Autophagy regulates lipolysis and cell survival through lipid droplet degradation in androgen-sensitive prostate cancer cells. Prostate 72: $1412-1422,2012$.
22. Li J, Zhao S, Zhou X, et al: Inhibition of lipolysis by mercaptoacetate and etomoxir specifically sensitize drug-resistant lung adenocarcinoma cell to paclitaxel. PloS One 8: e74623, 2013.

23. Hunt MC and Alexson SE: The role acyl-CoA thioesterases play in mediating intracellular lipid metabolism. Prog Lipid Res 41: 99-130, 2002.

24. Hunt MC, Yamada J, Maltais LJ, Wright MW, Podesta EJ and Alexson SE: A revised nomenclature for mammalian acyl-CoA thioesterases/hydrolases. J Lipid Res 46: 2029-2032, 2005.

25. Hunt MC, Siponen MI and Alexson SE: The emerging role of acyl-CoA thioesterases and acyltransferases in regulating peroxisomal lipid metabolism. Biochim Biophys Acta 1822: 1397-1410, 2012

26. Ellis JM, Wong GW and Wolfgang MJ: Acyl Coenzyme A thioesterase 7 regulates neuronal fatty acid metabolism to prevent neurotoxicity. Mol Cell Biol 33: 1869-1882, 2013.

27. Han S and Cohen DE: Functional characterization of thioesterase superfamily member $1 /$ Acyl-CoA thioesterase 11: implications for metabolic regulation. J Lipid Res 53: 2620-2631, 2012.

28. Kang HW, Ozdemir C, Kawano Y, et al: Thioesterase superfamily member 2/Acyl-CoA thioesterase 13 (Them2/Acot13) regulates adaptive thermogenesis in mice. J Biol Chem 288: 33376-33386, 2013.

29. Kang HW, Niepel MW, Han S, Kawano Y and Cohen DE: Thioesterase superfamily member $2 /$ acyl-CoA thioesterase 13 (Them2/Acot13) regulates hepatic lipid and glucose metabolism. FASEB J 26: 2209-2221, 2012.

30. Ramakrishna M, Williams LH, Boyle SE, et al: Identification of candidate growth promoting genes in ovarian cancer through integrated copy number and expression analysis. PloS One 5: e9983, 2010.

31. Jung WY, Kim YH, Ryu YJ, et al: Acyl-CoA thioesterase 8 is a specific protein related to nodal metastasis and prognosis of lung adenocarcinoma. Pathol Res Pract 209: 276-283, 2013.

32. Edgar R, Domrachev M and Lash AE: Gene Expression Omnibus: NCBI gene expression and hybridization array data repository. Nucleic Acids Res 30: 207-210, 2002.

33. Roessler S, Long EL, Budhu A, et al: Integrative genomic identification of genes on 8p associated with hepatocellular carcinoma progression and patient survival. Gastroenterology 142: 957-966, 2012.

34. Lee JS, Chu IS, Mikaelyan A, et al: Application of comparative functional genomics to identify best-fit mouse models to study human cancer. Nat Genet 36: 1306-1311, 2004

35. Tung EK, Mak CK, Fatima S, et al: Clinicopathological and prognostic significance of serum and tissue Dickkopf-1 levels in human hepatocellular carcinoma. Liver Int 31: 1494-1504, 2011.

36. Hoshida Y, Villanueva A, Kobayashi M, et al: Gene expression in fixed tissues and outcome in hepatocellular carcinoma. N Engl J Med 359: 1995-2004, 2008.

37. Lu TJ, Lai WY, Huang CY, et al: Inhibition of cell migration by autophosphorylated mammalian sterile 20-like kinase 3 (MST3) involves paxillin and protein-tyrosine phosphatase-PEST. J Biol Chem 281: 38405-38417, 2006

38. Maloberti PM, Duarte AB, Orlando UD, et al: Functional interaction between acyl-CoA synthetase 4 , lipooxygenases and cyclooxygenase- 2 in the aggressive phenotype of breast cancer cells. PloS One 5: e15540, 2010.

39. Sun Z, Asmann YW, Kalari KR, et al: Integrated analysis of gene expression, $\mathrm{CpG}$ island methylation, and gene copy number in breast cancer cells by deep sequencing. PloS One 6: e17490, 2011.

40. Joshi-Barve S, Barve SS, Amancherla K, et al: Palmitic acid induces production of proinflammatory cytokine interleukin- 8 from hepatocytes. Hepatology 46: 823-830, 2007.

41. Kurahashi N, Inoue M, Iwasaki M, Sasazuki S and Tsugane S: Dairy product, saturated fatty acid, and calcium intake and prostate cancer in a prospective cohort of Japanese men. Cancer Epidemiol Biomarkers Prev 17: 930-937, 2008. 